\title{
In the Office and In-Between: The Role of Panel Management in Primary Care
}

\author{
Catherine P. Kaminetzky, MD, MPH', and Karin M. Nelson, MD, MSHS ${ }^{2,3}$ \\ ${ }^{1}$ Center for Education and Development, VA Puget Sound Health Care System, Seattle, WA, USA; ${ }^{2}$ Division of General Internal Medicine, University \\ of Washington School of Medicine, Seattle, WA, USA; ${ }^{3}$ VA Health Services Research and Development, VA Puget Sound Health Care System, \\ Seattle, WA, USA.
}

J Gen Intern Med 30(7):876-7

DOI: $10.1007 / \mathrm{s} 11606-015-3310-\mathrm{x}$

(C) Society of General Internal Medicine 2015

$\mathrm{P}$ anel management is defined as a "set of tools and processes for population care that are applied systematically at the level of a primary care panel." ${ }^{1,2}$ Panel management typically involves non-physicians who utilize chronic disease registries, electronic health records, and data support tools to identify missed opportunities for unmet preventive care and chronic disease care, and who communicate recommendations from the provider to the patient. ${ }^{2}$ Teams may utilize panel management to reach out to every patient at each visit, called in-reach, or to address unmet needs between patient visits, called outreach. $^{1,2}$

Panel management has been successfully implemented in patient-centered medical homes, often in the context of a teambased model of care. ${ }^{3}$ The use of medical assistants or nurses to conduct panel management has been associated with improved care process outcomes such as improved rates of vaccination, health care proxy designation, and bone density screening in the care of elderly patients. ${ }^{4}$ Other studies have shown improvements in clinical outcomes, but panel management in these settings was part of a complex intervention, making it difficult to determine to what degree improvements can be attributed to panel management alone. ${ }^{3,5}$

Successful panel management programs need to be supported by computerized clinical support systems that provide relevant care reminders at the point of care, flexible data registries, and performance feedback. Implementation of electronic panel support tools with such capabilities have been associated with enhanced chronic disease management. ${ }^{6}$ It is not known whether these systems are cost-effective, ${ }^{7}$ and they may be applicable only to large integrated health systems or accountable care organizations (ACOs). ${ }^{6,7}$ Even in systems with a panel management support tool, there are barriers to its utilization, such as insufficient time, competing demands, and suboptimal staffing. Facilitators of panel management include strong team roles, leadership support and training for tool implementation, and dedicated time for use. ${ }^{8}$

Published online April 8, 2015
In this issue, Schwartz et al. ${ }^{9}$ report on the effects of adding nonclinical staff to perform panel management in the care of patients with hypertension and/or who smoked. In an 8-month pragmatic cluster-randomized controlled trial at two Veterans Administration (VA) primary care sites, primary care teams in the intervention groups received either panel management assistant (PMA) support or PMA support plus targeted panel management education, while control providers received monthly lists of current smokers and patients with uncontrolled hypertension. PMAs were non-clinicians with undergraduate degrees who received training in clinical issues associated with hypertension and smoking, panel management skills, electronic and administrative tools including the VA electronic health record, motivational interviewing, and Brief Action Planning, and they received panel management toolkits. PMAs were embedded within primary care teams, and they systematically reviewed panel data to identify patients with gaps in care, proposed interventions to primary care teams in biweekly meetings, and subsequently contacted patients by mail and phone.

Primary outcomes included mean systolic and diastolic blood pressure at baseline and post-intervention as well as smokers' self-reported quit attempts. Secondary clinical outcomes included process measures such as patient participation in the VA's weight management program (MOVE!) and homebased electronic blood pressure monitoring program (Telehealth), and the number of prescriptions for nicotine replacement therapy (NRT). Staff surveys measured attitudes toward panel management, panel management training, and working with PMAs. PMAs measured team meeting frequency, panel management strategies used, patient populations targeted for interventions, outreach performed, and number of patients reached. Originally designed as a 12-month study, initiation of the trial was delayed due to the VA's national implementation of a patient-centered medical home model, the Patient Aligned Care Team (PACT), and the study ended early as a result of a natural disaster that closed one of its participating sites.

At the end of 8 months, the group found no difference in reductions in blood pressure or rates of smoking across the three arms. Intervention patients were more likely to receive NRT prescriptions and enroll in MOVE! or Telehealth. The authors report a dose effect, with better blood pressure control 
for intervention teams that met more frequently with their PMA. For all groups, the rate of staff reporting sufficient panel management training or education increased from $26 \%$ to $64 \%$ post-intervention, with no differences seen across study arms, either suggesting that contamination occurred as a result of the study or as a result of implementation of PACT and its associated training. Staff reports about the intervention were mixed: although most found the PMA useful, one-quarter reported that the PMA took too much time, and only $40 \%$ said they would continue panel management after the intervention, suggesting barriers to implementation without the additional staff.

There are several reasons why the authors may not have found a treatment effect. With the shortened duration of the study, it is not surprising that clinical outcomes, and even many processes of care, did not differ across the study arms. Furthermore, PMAs may not have had sufficient time or interaction with patients to establish the trusting relationship needed to effect important and lasting behavioral changes, since patients' perceptions of being "known as a person" by their providers have been associated with greater adherence to medication regimens. ${ }^{10}$ In addition, the VA has high rates of hypertension control compared to other clinical systems, and this was true in the current study: $78 \%$ of hypertensive patients had controlled blood pressure at baseline, compared to $50 \%$ in the United States. ${ }^{11}$ Such high quality of care may have created a ceiling effect that was difficult to overcome. Indeed, previous positive studies of panel management were performed in sites with much lower quality indicators at baseline. ${ }^{4}$ Finally, the timing of the VA's implementation of PACT represents another difficulty in interpreting the results, leading one to wonder whether this study, in fact, measured the results of adding PMAs to fully staffed patient-centered medical homes.

More study is needed to understand whether panel management can improve health outcomes, at what dose and in which patients. Other potential outcomes deserve study as well. Could redistributing clinical work from primary care providers to other staff increase work capacity in primary care settings? ${ }^{12}$ Does panel management affect primary care practitioner satisfaction or rates of burnout, and if so, in what direction? As the nation's largest health care system, the VA has already invested in panel management by embedding nurse care managers in primary care to perform outreach and chronic care management using robust data systems, expanding staffing models to include support staff who collaborate in in-reach and outreach activities, and training providers and staff in their new roles as team members. How and whether smaller health care systems should implement panel management will hopefully become more clear with further study.

Conflict of Interest: The authors have no conflicts of interest to report.

Corresponding Author: Catherine P. Kaminetzky, MD, MPH; Center for Education and DevelopmentVA Puget Sound Health Care System, 1660 South Columbian Way, Seattle, WA 98108, USA (e-mail: catherine.kaminetzky@va.gov).

\section{REFERENCE}

1. Neuwirth EE, Schmittdiel JA, Tallman K, Bellows J. Understanding panel management: a comparative study of an emerging approach to population care. Perm J. 2007;11(3):12-20.

2. Chen EH, Bodenheimer T. Improving population health through teambased panel management: comment on "Electronic medical record reminders and panel management to improve primary care of elderly patients". Archives of internal medicine. 2011;171(17):1558-1559.

3. Reid RJ, Coleman $\mathbf{K}$, Johnson EA, et al. The Group Health medical home at year two: cost savings, higher patient satisfaction, and less burnout for providers. Health Aff (Millwood). 2010;29(5):835-843.

4. Loo TS, Davis RB, Lipsitz LA, et al. Electronic medical record reminders and panel management to improve primary care of elderly patients. Archives of internal medicine. 2011;171(17):1552-1558.

5. Chuang E, Ganti V, Alvi A, Yandrapu H, Dalal M. Implementing panel management for hypertension in a low-income, urban, primary care setting. J Prim Care Community Health. 2014;5(1):61-66.

6. Zhou YY, Unitan R, Wang JJ, et al. Improving population care with an integrated electronic panel support tool. Popul Health Manag. $2011 ; 14(1): 3-9$.

7. Garg AX, Adhikari NK, McDonald H, et al. Effects of computerized clinical decision support systems on practitioner performance and patient outcomes: a systematic review. Jama. 2005;293(10):1223-1238.

8. Feldstein AC, Schneider JL, Unitan R, et al. Health care worker perspectives inform optimization of patient panel-support tools: a qualitative study. Popul Health Manag. 2013;16(2):107-119.

9. Schwartz MD, Jensen A, Wang B, et al. Panel Management to Improve Smoking and Hypertension Outcomes by VA Primary Care Teams: A Cluster-Randomized Controlled Trial. J Gen Intern Med. Feb 10 2015. [SPI 3204]

10. Beach MC, Keruly J, Moore RD. Is the quality of the patient-provider relationship associated with better adherence and health outcomes for patients with HIV? J Gen Intern Med. 2006;21(6):661-665.

11. Egan BM, Zhao Y, Axon RN. US trends in prevalence, awareness, treatment, and control of hypertension, 1988-2008. Jama. 2010;303(20):2043-2050.

12. Bodenheimer TS, Smith MD. Primary care: proposed solutions to the physician shortage without training more physicians. Health Aff (Millwood). 2013;32(11):1881-1886. 